\title{
Suppression of Natural Killer (NK) Cell Activity of Spleen Cells by Thymocytes ${ }^{1}$
}

\author{
Madhavan P. N. Nair, ${ }^{2}$ Stanley A. Schwartz, ${ }^{*}$ Gabriel Fernandes, \\ Rajendra Pahwa, Susumu IKehara, and Robert A. Good \\ Memorial Sloan-Kettering Cancer Center, 1275 York Avenue, New York, New York 10021, and \\ * Department of Pediatrics, The University of Michigan, Ann Arbor, Michigan 48109
}

Received December 31, 1979; accepted May 28, 1980

\begin{abstract}
The in vitro influence of thymus cells on natural killer cell activity of spleen cells against prelabeled target cells (YAC-I and RL $\$ 1$ ) has been studied in syngeneic as well as in allogeneic murine models. In mixing experiments to demonstrate suppression, total thymocytes have been found to have no effect on NK activity of syngeneic or allogeneic spleen cells. Among several thymocyte fractions separated by velocity sedimentation, a relatively faster sedimenting fraction showed remarkable suppression of NK activity by spleen cells against two target cells. The suppressive effect of this particular fraction on NK activity was demonstrated to be proportional to the cell dose. The suppressive function was resistant to irradiation at 1000 or $2000 \mathrm{rad}$ administered in vitro and was not restricted by the major histocompatibility complex. Moreover, the thymocyte fraction which induced suppression was not sensitive to NK-mediated cytolysis by syngeneic spleen cells. The suppression of NK cytolysis in vitro by certain subpopulations of thymocytes as observed in the present studies may be consistent with a role for the thymus in regulating NK activity in vivo.
\end{abstract}

\section{INTRODUCTION}

The regulatory role of thymus-derived $(\mathrm{T})$ cells has recieved considerable attention because of their potential acitvity in either amplifying or suppressing antibody synthesis, graft rejection, and generation of cytotoxic cells to alloantigens (1-4). In studies with thymocytes, Moiser and Johnson (5) had shown suppression of plaque-forming activity of adult spleen cells by neonatal thymocytes. Moticka (6) demonstrated both a suppressive influence of thymocytes on normal lymphocyte proliferative responses to PHA and that thymocytes inhibit the GVH response in transfer experiments using a chicken model.

Recently natural killer $(\mathrm{NK})^{3}$ cells have been gaining much attention because

${ }^{1}$ Aided by grants from the USPHS: NS-11457, AI-11843, AI-16216, CA-08748, CA-17404, AG 00541, DE-02731; The Fund for the Advanced Study of Cancer, Richard Molin Memorial Foundation, Coleman Laboratory, the Special Projects Committee MSKCC, and the Dental Research Institute Associate Member Program of the University of Michigan.

${ }^{2}$ Present address: Department of Pediatrics, The University of Michigan, Ann Arbor, Mich. 48109. To whom all correspondence and reprint requests are to be directed.

${ }^{3}$ Abbreviations used: $\mathrm{B} 6, \mathrm{C} 57 \mathrm{BL} / 6 \mathrm{~J}$ mouse strain; effector cells, spleen cells depleted of nylon wooladherent cells and monocyte/macrophages with carbonyl iron; E:T, effector to target; FCS, fetal calf serum; H-2, murine major histocompatibility complex; NK, natural Killer; RLsI, radiation-induced leukemia in BALB/c mouse strain; S:E, suppressor to effector; TdT, terminal deoxynucleotidyl transferase; YAC-I, T-cell lymphoma induced by Moloney virus in A/sm mouse strain. 
of a possible role in defense against tumor cells as well as in maintaining regulation of lymphoid cell reactions. Nunn et al. (7) and Hansson et al. (8) recently reported that a wide range of malignant and nontransformed cells including thymocytes from different strains of mice were sensitive to low levels of NK-mediated cytolysis, thereby implying that NK cells may be involved in potential homeostatic control mechanisms. If NK activity is important in self-regulation of lymphoid cell functions it seems likely that differentiation, maturation, and function of NK cells would also be subject to regulation. The finding of high NK activity in nude and neonatally thymectomized mice (9) suggests that thymocytes or cells under thymic influence might exert a regulatory influence on NK cells.

In the present study, we have examined the effect of different thymus cell subpopulations fractionated by velocity sedimentation on the NK activity of spleen cells against prelabeled YAC-I and RLố I target cells in vitro. We report suppression of NK activity of effector spleen cells against both target cells by a pooled fraction of syngeneic thymocytes, separated by velocity sedimentation. Cells larger or smaller than the active subpopulation did not suppress NK activity of spleen cells against either target cells. The suppressive function on the effector cells of the active fraction was dose dependent and not restricted by the major histocompatibility complex. The thymocyte fraction which showed suppression was not itself susceptible to NK-mediated lysis by syngeneic spleen cells. The results suggest that suppression of NK cytolysis by thymus cells in vitro is a manifestation of a thymicdependent regulatory mechanism modulating the level of NK activity in vivo.

\section{MATERIALS AND METHODS}

Mice. C57Bl/6J(B6) and $\mathrm{C} 3 \mathrm{H} / \mathrm{HeJ}(\mathrm{C} 3 \mathrm{H})$ mice purchased from Jackson Laboratory, Bar Harbor, Maine, were employed as a source of spleen and thymus cells. All mice were used at 5 to 8 weeks of age.

Preparation of effector cells. Spleen cells were collected in RPMI 1640 medium (Grand Island Biological Co., Grand Island, N.Y.) supplemented with $10 \%$ fetal calf serum (FCS). The cells were released by gently teasing the spleens or thymuses in cold medium. Erythrocytes were lysed by treatment with isotonic ammonium chloride $(0.83 \%)$ in $0.016 M$ Tris buffer $(\mathrm{pH} 7.2)$ for 2 min at $4{ }^{\circ} \mathrm{C}$ and the cells were then washed three times in complete medium. Finally the cells were resuspended in complete medium to a concentration of $2 \times 10^{8}$ cells $/ \mathrm{ml}$. The cells were then passed through a nylon wool column to remove the adherent cells as described by Handwerger and Schwartz (10). Monocytes/macrophages were removed by the carbonyl iron method as described elsewhere (11).

Preparation of thymus cells. Thymuses were removed aseptically, minced, and gently passed through a fine mesh stainless steel sieve into phosphate-buffered saline (PBS). Cell aggregates were disrupted further by repeated aspiration with a Pasteur pipet. After removing large cell clumps and debris, the cells were passed through the mesh and washed three times with PBS at $4^{\circ} \mathrm{C}$. Cell viability was 90 to $95 \%$ as determined by trypan blue dye exclusion. The cells were then separated by velocity sedimentation at unit gravity at $4^{\circ} \mathrm{C}$ according to a modification of the method of Miller (12). Details of the separation procedure have been described elsewhere (13). Cells were allowed to sediment at $4^{\circ} \mathrm{C}$ for $4 \mathrm{hr}$ and then collected in $35-\mathrm{ml}$ fractions after discarding an initial $250 \mathrm{ml}$. Thirty fractions were collected. The cell concentration in each fraction was determined in a hemocytometer; ad- 
jacent fractions were pooled when necessary and then washed twice with PBS. Total cell recovery was 70 to $85 \%$, and cell viability was 90 to $95 \%$ as measured by trypan blue dye exclusion.

Assay for NK cell activity. The NK activity was determined in a direct ${ }^{51} \mathrm{Cr}$ release assay as previously described (14). Briefly a fixed number of viable spleen cells in complete medium was prepared in varying dilutions to give $50: 1,25: 1$, and 12.5:1 effector to target (E:T) cell ratios. The spleen cells were added in triplicate to ${ }^{51} \mathrm{Cr}$-labeled target cells in a final volume of $0.2 \mathrm{ml}$ in $\mathrm{V}$-bottom microtiter plates (Falcon No. 3041, Rockville, Md.) and following $40 \mathrm{~g}$ centrifugation for $2 \mathrm{~min}$ incubated at $37^{\circ} \mathrm{C}$ in $5 \% \mathrm{CO}_{2}$ in air atmosphere for $4 \mathrm{hr}$. In mixing assays, to measure suppressor activity, total thymocytes or fractionated subpopulations of thymocytes were added separately to a mixture of effector and target cells. In mixing experiments, the E:T cell ratio was maintained at 50:1 whereas the suppressor to effector $(S: E)$ cell ratio ranged from 1:1, 2:1, and 3:1. All plates were treated exactly as described for the cytotoxicity assay. Percentage cytotoxicity was calculated as follows:

$$
\text { Percentage lysis }=\frac{\text { experimental release }- \text { spontaneous release }}{\text { maximal release }(\text { Triton } x-100)-\text { spontaneous release }} \times 100 .
$$

Tumor cells. RLôI, a cell line derived from radiation-induced T-cell leukemia in BALB/c mice (15) and YAC-I, a T-cell lymphoma induced by Moloney virus in $\mathrm{A} / \mathrm{Sn}$ mice obtained through the courtesy of O. Stutman, Sloan-Kettering Institute, New York, were used as target cells. All the tumor target cells were maintained in our laboratory by serial passage in vitro in RPMI 1640 medium (Gibco) supplemented with $10 \%$ FCS, glutamine, and antibiotics. Target cells were labeled with ${ }^{51} \mathrm{Cr}$ as sodium chromate (Radiochemical Center, Amersham, England) as described elsewhere (14).

Irradiation of cells. Total thymocytes or their subfractions were irradiated with 1000 or 2000 rad of $\gamma$ irradiation from a Cesium-137 source at a rate of $125 \mathrm{rad} /$ min. The irradiated cells were $>98 \%$ viable as judged by trypan blue dye exclusion.

\section{RESULTS}

\section{Suppression of NK Activity of Spleen Cells by Syngeneic Thymus Fractions}

In view of the fact, that nude mice or thymectomized mice showed high levels of NK activity (9), the in vitro influence of thymus cells on NK activity of syngeneic spleen cells was studied against prelabeled YAC-I and RLỏI target cells (Fig. 1). In general, cytolysis, as measured by release of ${ }^{51} \mathrm{C}$, was higher against YAC-I target cells than against RLôI cells. Total thymocytes or thymocytes fractionated by velocity sedimentation by themselves did not show cytolytic activity against either YAC-I or RLỏI target cells (data not presented). Spleen cells, from 5- to 8-week-old B6 mice, depleted of nylon wool-adherent cells and monocytes/macrophages showed 20 and $11 \%$ cytotoxicity against YAC-I and RLôI target, respectively. To detect the effect of unfractionated thymocytes or thymocyte fractions on effector cell function in NK activity, total thymocytes or their fractions were added to functional NK cells from young B6 mice and radiolabeled target cells. The results demonstrate that the mixture containing total thymocytes does not show a suppressive effect on NK activity of syngeneic spleen cells against YACI ( $18 \%$ cytotoxicity) or against RLóI ( $10 \%$ cytotoxicity) target cells. Pooled thymocytes from fractions 15-18 showed remarkable suppression of NK activity of 


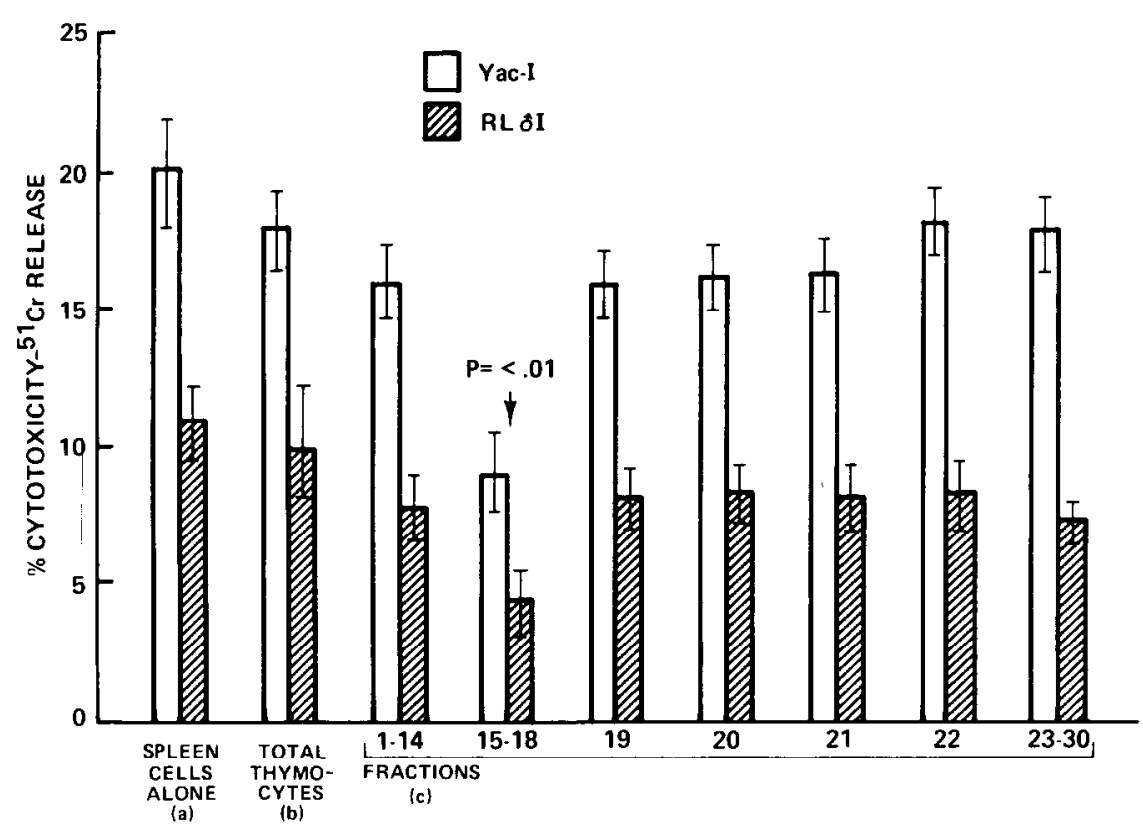

FIG. 1. Suppression of natural killer cell activity of young B6 spleen cells by autologous thymocytes fractionated by velocity sedimentation. Effector cells were depleted of nylon wool-adherent cells and monocyte/macrophages. Effector to target cell ratios (E:T) of 50:1, 25:1, and 12.5:1 were used in the experiments and dose response cytotoxicity was obtained, but only results of the 50:1 E:T ratio are shown. (ㅁ)Percentage cytotoxicity against YAC-I. (㑮)Percentage cytotoxicity against RLðI cells. In the suppressor assay, $5 \times 10^{5}$ thymocytes or their fractions were added separately to a mixture of $5 \times 10^{5}$ effector cells and $1 \times 10^{4}$ prelabeled target cells and following $4 \mathrm{hr}$ incubation, percentage cytotoxicity was measured as shown under Materials and Methods. A constant suppressor:effector (S:E) cell ratio of $1: 1$ has been used. Results are expressed as mean percentage \pm SE of direct cytotoxicity to target cells in triplicate culture of one representative experiment. Five separate experiments gave similar results. (a) Percentage cytotoxicity by spleen cells alone; (b) percentage cytotoxicity by spleen cells in presence of total thymocytes; (c) percentage cytotoxicity by spleen cells in presence of different thymocyte fractions. Pooled thymocyte fraction 15-18 was the only fraction that showed significant suppression of NK activity of autologous splecn cells against both YAC-I and RL\&I targets, $P<0.01$.

syngeneic spleen cells against YAC-I (9.0\% cytotoxicity) or RLỏI (4.5\% cytotoxicity) targets compared to lack of suppression observed with total thymocytes or earlier thymocyte fractions $1-14$ or later fractions $19,20,21,22$, or $23-30$. The cytotoxicity exerted by spleen cells in cultures containing thymocytes of fractions 1-14 were 16.0 and $8.0 \%$, respectively, against YAC-I and RL $\delta$ I target cells. Also no suppression of cytotoxicity of spleen cells was obtained by any of the later fractions against either YAC-I or RLốI target cells. The results indicate a significant suppressive effect $(P=<0.01)$ on NK activity of spleen cells by syngeneic thymocytes from fractions $15-18$ separated by velocity sedimentation.

\section{Suppression of NK Activity is Mediated across the Major Histocompatibility Barrier}

To test whether the suppressive action can be mediated when thymocytes and spleen cells differ at the major histocompatibility complex, thymocytes and thymocyte fractions from $\mathrm{B} 6\left(\mathrm{H} 2^{\mathrm{b}}\right)$ mice were mixed with functional splenic NK cells from $\mathrm{C} 3 \mathrm{H}\left(\mathrm{H}-2^{\mathrm{k}}\right)$ mice and prelabeled YAC-I and RLố target cells. Representative 
data are presented in Fig. 2. C3H spleen cells depleted of nylon wool-adherent cells and of monocyte/macrophages by the carbonyl iron method showed 22.0 and $11.8 \%$ cytotoxicity against YAC-I and RLôI targets, respectively. In mixing experiments to measure allogeneic suppression, the mixture containing total thymocytes from B6 did not show any suppressive effect on $\mathrm{NK}$ activity of $\mathrm{C} 3 \mathrm{H}$ spleen cells against YAC-I (19.0\% cytotoxicity) and RLôI (11.0\% cytotoxicity) target cells. Pooled thymocytes from fractions 15-18 showed significant suppression of NK activity of allogeneic $\mathrm{C} 3 \mathrm{H}$ spleen cells against YAC-I (7.0\% cytotoxicity) and RLỏ ( $4.0 \%$ cytotoxicity) target cells, compared to lack of suppression obtained by total thymocytes or other fractions of thymocytes $(P=<0.01)$. Effector cells plus added thymocyte fractions 1-14 did not show any significantly decreased cytotoxicity against YAC-I and RL $\mathbf{I}$ targets. Thymocyte fractions 19, 20, 21, 22, and 23-30 also did not cause suppression of cytotoxicity of spleen cells against either target cell. The results thus demonstrate that suppression of NK activity is mediated by thymocyte fractions $15-18$ and is active across the major histocompatibility barrier and as described above for syngeneic cells, no suppression is manifested by thymocyte fractions other than 15-18.

\section{Dose Response Effect of Suppressor Thymocytes on NK Activity of Syngeneic Spleen Cells}

The results of dose response analysis of the suppressive effect of B6 thymocytes and their fractions on NK activity of autologous spleen cells against prelabeled

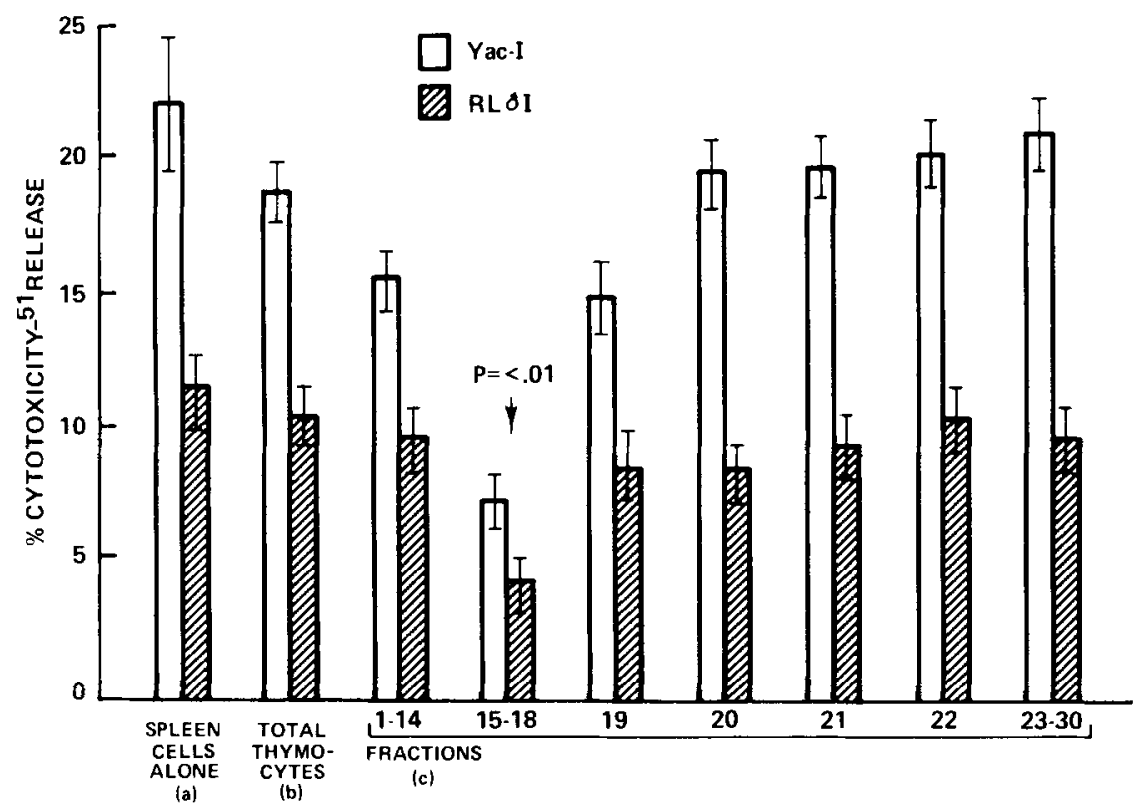

FIG. 2. Suppression of natural killer cell activity of young $\mathrm{C} 3 \mathrm{H}\left(\mathrm{H}-2^{\mathrm{k}}\right)$ spleen cells by allogeneic $\mathrm{B} 6$ $\left(\mathrm{H}-2^{b}\right)$ thymocyte fraction. See legend of Fig. 1 for E:T and $S: E$ ratios used and for experimental details. Results are expressed as mean \pm SE of percentage direct cytotoxicity to target cells in triplicate cultures of a representative experiment; five separate experiments gave similar results. (a) Percentage cytotoxicity by $\mathrm{C} 3 \mathrm{H}$ spleen cells alone; (b) percentage cytotoxicity by $\mathrm{C} 3 \mathrm{H}$ spleen cells in presence of total thymocytes from B6; (c) percentage cytotoxicity by $\mathrm{C} 3 \mathrm{H}$ spleen cells in presence of different fractions of B6 thymocytes. Results show significant suppression of $\mathrm{NK}$ activity of $\mathrm{C} 3 \mathrm{H}$ spleen cells was mediated by the velocity-sedimented fractions $15-18$ of allogeneic (B6) thymocytes $(P<0.01)$. 
YAC-I target cells are shown in Fig. 3. The suppressive effect of pooled thymocyte fractions 1518 on NK activity of spleen cells was noticeable at a S:E cell ratio of $1: 1$. At an $S: E$ ratio of $1: 1$, the effector cells containing the active thymocyte pool (fractions 15-18) showed significant lowering of NK activity. NK activity of effector cells was decreased still further, when pooled thymocyte fractions 15-18 were added at thymocyte to effector ratios of $2: 1$ and 3:1. Total thymocytes or pooled fractions of thymocytes, numbered 1-14 or 19-30, had little suppressive effect on the NK activity of effector spleen cells at any thymocyte to effector cell ratio. These results demonstrate a cell dose-dependent suppressive effect of pooled thymocyte fractions $15-18$ on the NK activity of syngeneic effector cells against YAC-I target cells.

\section{Lack of Influence of Irradiation on Suppressive Effect of Thymocyte Fraction}

To examine the sensitivity of the suppressor thymocyte fraction to irradiation, pooled thymocyte fractions $15-18$ were irradiated at 1000 and $2000 \mathrm{rad}$ and their influence on the NK activity of effector cells against YAC-I target cells was studied. Representative data are shown in Fig. 4. Pooled thymocyte fractions 15-18, nonirradiated or irradiated at 1000 or 2000 rad, caused significant suppression of NK activity of autologous spleen cells against YAC-I target cells. No significant differences were observed in the suppression of NK activity of spleen cells against YAC-I target cells by irradiated suppressor cells versus nonirradiated suppressor cells at an S:E ratio of 1:1. The total thymocytes, irradiated at 1000 or $2000 \mathrm{rad}$ or nonirradiated total thymocytes did not show any suppressive effect on NK ac-

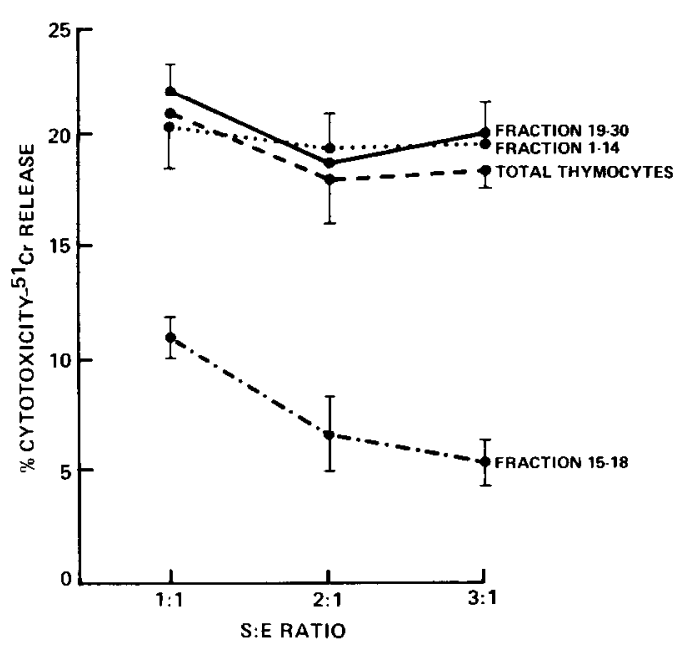

FIG. 3. Dose response effect of suppressor thymocytes on NK activity of autologous spleen cells of B6 mice. A constant E:T cell ratio of 50:1 was used. Prelabeled YAC-I cells were used as targets. S:E ratios of $1: 1,2: 1$, and 3:1 were employed. Results are expressed as mean percentage \pm SE of direct cytotoxicity to target cells in triplicate cultures in a representative experiment; four separate experiments gave similar results. Results show a significant dose response suppressive effect of thymocyte fraction 15-18 on NK activity of autologous effector cells against YAC-I target cells compared to that of total thymocytes or other pooled fractions of thymocytes. 
tivity of spleen cells against YAC-I target cells. These findings demonstrate that the suppressive action of thymocytes on NK activity was not sensitive to in vitro irradiation.

\section{The Thymocyte Fraction which Induced Suppression was Not Sensitive to NK- Mediated Cytolysis}

These experiments were designed to investigate whether suppression observed by thymocytes was due to cold target cell competitive inhibition of NK activity rather than actual suppression. Spleen cells, unfractionated thymocytes, and different thymocyte fractions from 5- to 8-week-old B6 mice were labeled with ${ }^{51} \mathrm{Cr}$ as detailed under Materials and Methods. Splenocytes from the same animals were passed over nylon wool and the recovered cells were used as effector cells. Results of a representative experiment are presented in Table 1. At an E:T ratio of 50:1 or 100:1, unseparated thymocytes or any of their fractions failed to show cytolysis by syngeneic spleen cells. No activity was observed against prelabeled spleen cells. The study thus revealed that thymocyte fractions 15-18 which showed significant suppression of NK activity of spleen cells did not contain antigenic determinants recognizable by the syngeneic NK cell and therefore suppression obtained in the present studies could not be attributed to cold target cell competitive inhibition, but rather to true suppression by thymocytes.

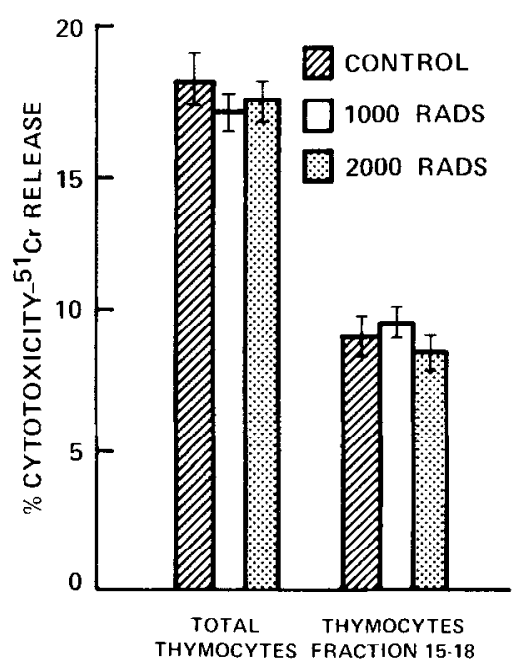

FIG. 4. Suppressive function of B6 thymocytes on NK activity of autologous spleen cells against YACI target cells was not irradiation sensitive. Total thymocytes and fraction 15-18 were irradiated in vitro at 1000 and $2000 \mathrm{rad}$. E:T ratio of 50:1 and $\mathrm{S}: \mathrm{E}$ ratio of $1: 1$ were used. Results are expresscd as mcan percentage \pm SE of direct cytotoxicity to target cells in triplicate culture of one respresentative exper-

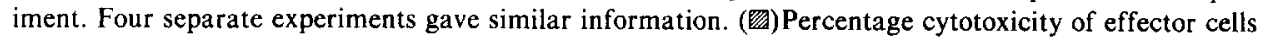
in presence of nonirradiated thymus fraction 15-18 or total thymocytes. (D)Percentage cytotoxicity of effector cells in presence of irradiated thymus fraction 15-18 or total thymocytes at 1000 rad. ( $\$$ ) Percentage cytotoxicity in presence of thymus fraction 15-18 or total thymocytes irradiated at 2000 rad. Results showed that thymocyte fraction 15-18-induced suppression of NK activity of spleen cells was not sensitive to 1000 or 2000 rad of $\gamma$ irradiation administered in vitro. 
TABLE 1

Cytotoxicity of Syngeneic Spleen Cells against Prelabeled Thymocytes and Their Various Fractions ${ }^{a}$

\begin{tabular}{llc} 
& \multicolumn{2}{c}{ Percentage cytotoxicity at } \\
& E:T ratio \\
\cline { 2 - 3 } Target cells & $50: 1$ & $100: 1$ \\
\hline Total thymocytes & 0.68 & 0.18 \\
Fractions 1-14 & 0.12 & 1.08 \\
Fractions 15-18 & 1.14 & 0.02 \\
Fraction 19 & 0.89 & 0.17 \\
Fraction 20 & 0.21 & 0.00 \\
Fraction 21 & 0.67 & 0.70 \\
Fraction 22 & 0.45 & 0.10 \\
Fractions 23-30 & 1.25 & 0.62 \\
Total spleen cells & 0.89 & 0.28 \\
\hline
\end{tabular}

\footnotetext{
${ }^{a}$ Spleen cells did not show any cytotoxicity against prelabeled thymocytes or their fractions. Total thymocytes and their various fractions were labeled with ${ }^{51} \mathrm{Cr}$ as described under Materials and Methods and used as target cells. Syngeneic spleen cells depleted of nylon wool-adherent cells and monocyte/ macrophages, were used as effector cells. Effector to target cell ratios of 50:1 and 100:1 were used. Results are expressed as mean percentage cytotoxicity to target cells in triplicate cultures of one representative experiment. Results show that unfractionated thymocytes or any of their subfractions are not sensitive to NK-mediated cytolysis by spleen cells.
}

\section{DISCUSSION}

Cells playing a regulatory role in the immune response have been widely studied and documented in murine systems. Most of the regulatory cells are thymocytes or are thymus-dependent cells. In the present studies, using cell mixing experiments to demonstrate inhibition, it was possible to show the presence of NK specific suppressor cells in a particular subpopulation of thymus cells. "Suppression" is operationally defined as the direct inhibition of cytolysis resulting from the addition of thymus cells or velocity sedimentation fractions to a mixture of functional NK effector cells and prelabeled target cells. No preincubation of the suppressor with the effector cells was used.

Among different thymocyte fractions separated by a velocity sedimentation method, pooled cells of fractions 15-18 sedimenting at unit gravity at a rate between 4 and $5 \mathrm{~mm} / \mathrm{hr}$ showed consistent suppression of NK activity of young syngeneic as well as allogeneic spleen cells against YAC-I and RLốl target cells, compared to earlier or later fractions or unseparated thymocytes. The suppression observed by pooled fractions 15-18 of thymocytes does not appear to be due to crowding, spacing, or dilution of effector cells, since effector cells mixed with equal numbers of total thymocytes or differentially sedimenting subpopulations showed no suppression of the NK activity of effector cells.

The suppressive functions were resistant to irradiation administered in vitro and in this characteristic are like the suppressor cells of spleen described by Cudkowicz and Hochman (16). Studies of other characteristic features of the pooled thymocyte fractions $15-18$ in our laboratory have shown these fractions to contain large lymphocytes that are nonspecific esterase negative and contain the highest content of 
terminal deoxynucleotidyl transferase (TdT) activity, a marker enzyme for precursor $T$ cells or immature thymocytes ${ }^{4.5}$.

Pazmino et al. (17) and Small et al. (18) have recently reported that bone marrow from $\mathrm{nu} / \mathrm{nu}$ Swiss mice and neonatally thymectomized C57BL/6 mice has a 10 -fold reduction in $\mathrm{TdT}$ activity. Interestingly, $\mathrm{nu} / \mathrm{nu}$ mice or neonatally thymectomized mice express a high percentage of NK cells (9) perhaps because NK specific suppressor cells presumably derived from the thymus are lacking in their marrow. This finding is consistent with our observation that NK specific suppressor cells appear to be TdT positive thymus-derived lymphocytes.

Small and Trainin (19) and Gabizon et al. (20) have demonstrated that tumorinhibiting $\mathrm{T}$ cells in the spleens of tumor-bearing mice are gradually overwhelmed by a dominating population of tumor enhancing $T$ cells as the tumor progresses. This change could be correlated with enlargement of the spleen and involution of thymus (20-22). Small et al. (18) and Umiel et al. (23) recently postulated that thymocytes of tumor-bearing animals might be released prematurely into the spleen and that they then might be involved in eclipse of antitumor activity and consequently enhancement of tumor growth. Such cells have been identified in spleen as TdT positive cells, a marker for thymus-derived cells. In the present report, pooled TdT-enriched thymocyte fractions $15-18$, separated by velocity sedimentation and capable of manifesting significant suppression of the NK activity of both syngeneic and allogeneic spleen cells, may be identical with the tumor enhancing T-cell subpopulation of tumor-bearing mice reported by Small et al. (18) and Umiel et al. (23). Further characterization of these NK specific suppressor cells with respect to their ontogeny, tissue distribution, migratory and regulatory functions, both in normal and pathological conditions remains to be done.

The NK suppressive fraction of thymocytes was not sensitive to lysis by autologous spleen cells, suggesting that the suppression obtained in the present studies was not due to cold target cell competitive inhibition of NK activity by thymus cells. In previous studies Nunn, et al. (7) reported that spleen cells from Balb/c nude mice showed NK activity against thymus cells from different strains of mice. However, allogeneic or syngeneic spleen cells failed to show any cytotoxic reactivity against prelabeled thymocytes. Our study in which we observed no reactivity with syngeneic spleen cells against prelabeled thymus cells is consistent with the findings of Nunn et al. In similar experiments Hansson et al. (8) recently observed NK sensitivity of thymocytes from various strains of mice with high or low NK activity. They have reported that NK sensitivity of thymocytes varied markedly with the age of the thymocyte donor, reaching a peak at less than 1-2 weeks of age and no such sensitivity of thymocytes to NK-mediated lysis was seen in thymocyte populations of mice more than 3 weeks old. They also reported an inverse correlation between age dependence of splenic NK activity and the sensitivity of thymocytes to NK-mediated lysis. Therefore our findings which show a lack of NK sensitivity of a thymocyte population to spleen cells in mice aged 5-8 weeks is consistent with

\footnotetext{
${ }^{4}$ R. N. Pahwa, M. J. Modak, T. McMorrow, S. Pahwa, G. Fernandes, and R. A. Good, Terminal deoxynucleotidyl transferase enzyme in thymus and bone marrow. I. Age associated decline of TdT in humans and mice. Cell. Immunol. 58, 39, 1981.

${ }^{5}$ S. Ikehara, R. N. Pahwa, D. G. Luszer, R. A. Good, and M. J. Modak, Adenosine 5'-triphosphate (ATP)-mediated stimulation and suppression of DNA synthesis. I. Characterization of ATP responding cells in mouse lymphoid organs. Submitted for publication.
} 
our previous observations of peak NK reactivity in spleen at that period (24). Thus it is an exciting possibility that a feedback type of homeostatic control mechanism exists between thymus and spleen cells as the mice age.

The present study suggests that suppression of NK cytolysis in vitro by certain fractions of thymocytes may reflect a capacity for immunoregulatory activity of similar subpopulations on NK activity in vivo. Further characterization of these suppressor thymocytes could have the following significance: (i) If NK cells are involved in immune surveillance of malignancies (25), then enhancing their effects in premalignant and malignant states by diminishing NK specific suppressor activities could have significant benefit in the prevention or treatment of cancer. (ii) Evidence indicates that the identity of the effector cells involved in resistance to marrow transplantation is similar to NK cells (26), thus a reciprocal clinical situation, where effector cells could be inhibited and suppressor cell activity enhanced might be capable of prolonging marrow allografts in the treatment of leukemias, lymphomas, and immunodeficiencies.

\section{ACKNOWLEDGMENTS}

The authors also gratefully acknowledge the skillful technical assistance of Glenda McFeeter, Fred Barreau, John A. Rossi, Honorata R. Peralta, and Judith Miller Sanchez. We wish to thank Ms. Donna Meyer for excellent secretarial assistance.

\section{REFERENCES}

1. Claman, H. N., Chaperon, E. A., and Triplett, R. F., J. Immunol. 97, 828, 1966.

2. Cantor, H., and Simpson, E., Eur. J. Immunol. 5, 330, 1975.

3. Rich, S. S., and Rich, R. R., J. Exp. Med. 140, $1588,1974$.

4. Gershon, R. K., Liebhaber, S., and Ryu, S., Immunology 26, 909, 1974.

5. Moiser, D. E., and Johnson, B. M., J. Exp. Med. 141, 216, 1975.

6. Moticka, E. J., J. Immunol. 119, 987, 1977.

7. Nunn, M. E., Herberman, R. B., and Holden, H. T., Int. J. Cancer 20, 381, 1977.

8. Hansson, M., Kiessling, R., Anderson, B., Karre, K., and Roder, J., Nature (London) 278, 174, 1979.

9. Herberman, R. B., Nunn, M. E., and Lavrin, D. H., Int. J. Cancer 16, 216, 1975.

10. Handwerger, B. S., and Schwartz, R. H., Transplantation 18, 544, 1974.

11. Lundgren, G., Zukoski, C. F., and Moller, G., Clin. Exp. Immunol. 3, 817, 1968.

12. Miller, R. G., In "Techniques in Biophysics and Cell Biology" (P. Paris and B. Smith, Eds.), Vol. I, p. 87. Wiley, London, 1973.

13. Pahwa, R., Pahwa, S., and Good, R. A., J. Clin. Invest. 64, 1632, 1979.

14. Fernandes, G., Nair, M., Onoe, K., Tanaka, T., Floyd, R., and Good, R. A., Proc. Nat. Acad. Sci. USA 76, 457, 1979.

15. Sendo, E., Aoki, T., Boyse, E. A., and Buafo, C. R., J. Nat. Cancer Inst. 55, 603, 1975.

16. Cudkowicz, G., and Hochman, P. S., Immunol. Rev. 44, 13, 1979.

17. Pazmino, N. H., McEwan, R. N., and Ihle, J. N., J. Immunol. 119, 494, 1977.

18. Small, M., Lasser-Weiss, M., and Daniel, V., J. Immunol. 123, 259, 1979.

19. Small, M., and Trainin, N., J. Immunol. 117, 292, 1976.

20. Gabizon, A., Small M., and Trainin, N., Int. J. Cancer 18, 813, 1976.

21. Treves, A. J., Cohen, I. R., Schechter, B., and Feldman, M., Ann. N.Y. Acad. Sci. 276, 165, 1976.

22. Siegler, R., and Koprowska, I., Cancer Res. 22, 1278, 1962.

23. Umiel, T., Linkner-Israeli, M., Itzchaki, M., Trainin, N., Reisner, Y., and Sharon, N., Cell Immunol. 37, 134, 1978.

24. Floyd, R., Nair, M., Fernandes, G., and Good, R. A., Fed. Proc. 39, 1979.

25. Santoli, D., and Kaprowski, H., Immunol. Rev. 44, 125, 1979.

26. Trentin, J. J., and Bennett, M., Transplant. Proc. 9, 1303, 1977. 